\section{Rechenschaftsbericht GRAT/TARMED}

Hans Heinrich Brunner

Sehr geehrte Damen und Herren Liebe Kolleginnen und Kollegen

Unter www.tarmed.ch (CUG) finden Sie eine Zusammenstellung aller Beschlüsse der Ärztekammer zu GRAT und TARMED; nicht enthalten sind die Beschlüsse der Ärztekammer vom 12. April 2001.

Die Beschlüsse und die Kommentare betreffend ihrer Umsetzung (oder Nichtumsetzung) sind als Rechenschaftsbericht des unterzeichneten Verhandlungsleiters in den Jahren 1988 bis 2001 zu betrachten. Die Ausführungen beziehen sich ausschliesslich auf Anträge und Beschlüsse der Ärztekammer; diese sind im Gegensatz zu den Beschlüssen der Präsidentenkonferenz verbindlich; letztere haben aus statutarischen Gründen rein konsultativen Charakter. Ergänzt wird dieser Rechenschaftsbericht durch nachfolgende grundsätzliche Ausführungen:

1. Das GRAT-Projekt wurde zur Revision des UV-/MV-/IV-Arzttarifs (UMIA) konzipiert und mit einem Projektkredit von ursprünglich Fr. 7000 000.-, später durch Zusatzkredite ergänzt, versehen. Das Projekt hätte 1995 abgeschlossen und der neue UMIA unterbreitet werden können (die ursprünglichen Zielvorgaben waren zum vorneherein unrealistisch). Diese Ressourcen waren viel zu knapp, wie ein Vergleich mit ausländischen Projekten zeigt; so hat die Schaffung des vergleichbaren cpt-4 in den USA mehr als US\$ 150 Millionen gekostet. Folge war, dass es bis 2000 nicht gelungen ist, eine personell wie infrastrukturell adäquate Projektorganisation generell wie auch seitens der FMH aufzubauen. Dies erklärt einen Teil der in Phasen notorischen Fehlleistungen wie auch den Umstand, dass der Unterzeichnete sich mit der Wahl zum Präsidenten nicht auf die strategische Ebene zurückziehen konnte.

2. Völlig unhaltbar wurde die Situation mit der Erweiterung des GRAT/INFRA-Projektes in den KVG-Bereich unter dem Namen TARMED. Damals - 1996 - hätte eine völlig neue Projektorganisation mit breiterer Mitarbeiterbasis und grösseren Finanzmitteln sowie einem strikten Ablauf- und Finanzmanagement aufgebaut werden müssen. An Stelle eines solchen neuen Projektes wurde die bestehende GRAT-Leitung durch Delegationen von KSK und H+ ergänzt; die GRATAL, später TARMED, war die strategische wie operationelle
Drehscheibe, die die gesamte Last des Projektes mit etwas vermehrten Finanzmitteln zu tragen hatte. Das in letzter Minute lancierte INFRA-Projekt war personell und infrastrukturell dermassen unterdotiert, dass das gesteckte Ziel - KostenDaten-Ermittlung in Spitälern - zum vorneherein nicht erreichbar war. Es muss im Gegenteil geradezu verwundern, dass unter diesen Voraussetzungen das vorliegende Ergebnis erarbeitet werden konnte.

3. Die gesamte Termin- und Ablaufplanung wurde ab 1996 durch das EDI bzw. seine Forderungen nach Vorlage einer einheitlichen Tarifstruktur bestimmt. Durch das EDI, dessen Finanzierungsbeitrag sich bis heute auf genau Fr. 0.00 beläuft. Im nachhinein muss das Eintreten der Vertragsparteien auf diese Forderungen als nahezu deletär bezeichnet werden, um so mehr als für diese Forderungen nicht Andeutungen einer gesetzlichen Grundlage bestehen; Qualität und Fertigungstiefe des Projektes haben dadurch massive Einbussen erlitten. Wiederum zeigt ein Vergleich mit dem Ausland, dass ähnliche Projekte ein Laufzeit von 8 bis 10 Jahren haben; die von einer Gruppe wohlbekannter Medienleute immer wieder orchestrierte Kampagne der politischen Behörden nach dem Motto: «Warum denn jetzt immer noch nicht?» entbehrt mithin auch einer sachlichen Grundlage und hätte mit anderem als speichelleckerischer Willfährigkeit beantwortet werden müssen. Die Mitwirkung der Verhandlungsdelegation in diesem wenig würdigen Unterfangen mag sich dadurch erklären, dass sich die Ärzteschaft seit Beginn der 90er Jahre unter massivem Druck der Politik und der Öffentlichkeit befand, die im ebenso stereotypen wie unbegründeten Vorwurf gipfelte, die Bremser und Betonierer im Gesundheitswesen zu sein.

4. Das GRAT-Projekt war ausserordentlich stark vom Willen zu einem konsensuellen Vorgehen geprägt; man wollte keine Basartarife mehr, man wollte aber damit auch keine Konfrontationsverhandlungen mehr, wo jeweils Behauptung gegen Behauptung steht. Dies hat sich in der ersten Phase, der Konstruktionsphase, als zweckmässig erwiesen ... und alle darüber hinweggetäuscht, dass Versicherer und Ärztinnen und Ärzte in Grundsatzfragen verschiedene Positionen einnehmen, ja einnehmen müssen. Leute, die sonst konstruktiv zusammenarbeiten und durchaus auch ein kameradschaftliches Verhältnis pflegen, sind nun nicht die Leute, die von einer Stunde auf die andere konfrontative Verhandlungen in diesen Grundsatzfragen führen können und sollen. Retrospektiv gesehen wäre es gescheiter gewesen, diese zwingend notwendigen konfrontativen Verhandlungen, z.B. bezüglich Vergleichseinkommen, Normarbeitszeit, Auslastung u.a.m., andere Leute führen zu lassen. 
5. Die für die Schweiz typische Verpflichtung zum Konsens um nahezu jeden Preis erklärt auch, weshalb gewisse seit längerer Zeit klar erkennbare Defizite des Projektes nicht klarer angesprochen wurden. Gemeint sind vor allem die Defizite in Tarifmodell und -mechanik, die jetzt im Rahmen des Projektes Reengineering/Redesign (RR) ausgemerzt bzw. behoben werden sollen. Erklärungen zuhanden des Protokolls der Ärztekammer waren hier sicher ungenügend; der Unterzeichnete hätte hier unter Androhung des Verhandlungsabbruchs Besserung erzwingen müssen.

6. Seit Mitte der 90er Jahre war erkennbar, dass die Abgeltung von operativen-interventionellen Leistungen generell, in gewissen Gebieten speziell ausgeprägt, inadäquat abgesenkt werden würde. Es war keineswegs so, dass die Verhandlungsdelegation dies nicht erkannt und in den ursächlichen Mechanismen nicht richtig analysiert hätte. Die Bemühungen, durch nachhaltiges Verhandeln im einzelnen eine Korrektur zu erreichen, waren nur teilweise erfolgreich. Konnten wohl auch nur teilweise erfolgreich sein, weil alle anderen Vertragsparteien diesem Anliegen im besten Fall ablehnend, in der Regel aber feindlich gegenüberstanden. Dies gilt vor allem für die Vertretung der Spitäler, die in den letzten Jahren nur noch versuchten, ein möglichst grosses Scherflein für die Spitalinfrastrukturabgeltung ins trockene zu bringen. Auch hier wäre ca. 1998/99 ein Verhandlungsabbruch richtig gewesen, der aber aus der Angst heraus nicht erfolgte, das EDI würde einen "eigenen Tarif" in Kraft setzen, eine Drohung, die, wie wir heute wissen, nichts anderes als warme Luft war.

7. Es war und ist keineswegs so, dass die "Basis», sprich die Fachgesellschaften, nicht in die Arbeiten einbezogen worden wären. Keines der bekannten verwandten Projekte hat in nur andeutungsweise vergleichbarer Weise diese Ärztegruppen einbezogen. Letztes eindrückliches Beispiel war die Erarbeitung des EBM in Deutschland, die die Fachgesellschaften im besten Fall auf der Tribüne mitverfolgen konnten. Es wäre eine Studie in sich wert, weshalb der Vorwurf des mangelnden Einbezugs mit konstanter Regelmässigkeit immer wieder erhoben wurde und wird. Das Resultat wäre wahrscheinlich ein Puzzle bestehend aus falschem Verständnis des Entwicklungsprozesses, zweifelsohne phasen- und teilweise insuffizientem Projektmanagement seitens der FMH-Delegation, Abstinenz bis Fundamentalopposition gewisser Fachgesellschaften sowie dem Umstand, dass das historische Bewusstsein nicht weniger Gruppierungen zeitlich, und dies meist im besten Falle, die Amtsdauer ihrer Würdenträger umfasst.

8. In den letzten 3 bis 4 Jahren bzw. mit dem Einbezug des KVG-Bereichs wurden die Arbeiten vor- erst unbemerkt immer mehr durch politische Vorgaben belastet, die wenig, immer mehr aber überhaupt nichts mehr mit diesen Arbeiten zu tun hatten und haben. Erinnert sei an die unselige Kostenneutralitätsforderung, die bar jeglicher gesetzlichen Grundlage nichts anderes verfolgte und z.T. verfolgt als die Einführung des Globalbudgets durch die Ärzteschaft in einem Akt der freiwilligen Autoexekution. Als nur noch provokant kann die Abstinenz eben dieser politischen Akteure bezeichnet werden, die notwendigen Rahmenbedingungen festzulegen bzw. offene Fragen zu klären. Wer dies als Hausaufgaben definiert, die er anschliessend so ernst nimmt wie der übliche Schüler die Hausaufgaben für den Gesangsunterricht, darf sich nicht wundern, wenn der Gesang dann sehr schrill und unharmonisch wird. Ob runde Tische 1 Minute nach zwölf in der Sache noch weiterbringen, muss und mag an dieser Stelle offen bleiben. Sollten sie Anlass für eine weitere politische Fremdbestimmung und -verwendung bis hin zu Wahlmachinationen sein, kann schon heute das Scheitern des gesamten TARMED-Unternehmens im KVG-Bereich in Aussicht gestellt werden.

9. Ein ungelöstes Problem stellt im besonderen auch die Position der Preisüberwachung (PüW) dar. Sie wird sich bei der Einführung der kantonalen Tarife wieder zu Worte melden, sicher nicht im Sinne der Ärzteschaft. Diese sichere, in Inhalt und Auswirkungen nicht abschätzbare Intervention stellt für das Projekt eine der grössten Belastungen dar. Es kann trotz aktuell sehr einvernehmlichen Klimas in den Verhandlungen mit dem KSK keineswegs ausgeschlossen werden, dass nach $\mathrm{Ab}$ schluss der Verhandlungen die PüW das gesamte Verhandlungsresultat zu Makulatur machen und die Versicherer, die die PüW schon früher mit aller aktiven Unterstützung ihrerseits in ihrem Sinne agieren sahen, sich in pharisäerhafter Scheinheiligkeit wollüstig dem Diktat der PüW unterwerfen würden.

10. Nach Auffassung des Unterzeichneten hat sich die Verhandlungsposition der FMH gegenüber dem Tiefpunkt 1998 in den letzten 1.5 Jahren sukzessive verbessert. Würde das Paket, wie es der ÄK am 4.5.01 vorliegt, nicht akzeptiert werden, wird sich die Situation wieder Schritt für Schritt verschlechtern. Daran können auf lange Sicht auch massive politische Aktionen nichts ändern. $\mathrm{Zu}$ berücksichtigen ist hier, dass in den Augen der Öffentlichkeit bzw. der Politiker die FMH für eine handlungsfähige, kooperationsfähige Ärzteschaft steht. Würde in der ÄK eine Haltung in dem Sinne obsiegen, die Politiker sollten die Tarife schlussendlich gegen den Widerstand der Ärzteschaft einführen, würde dies die FMH nicht nur aller weiteren Einflussmöglichkeiten im Bereiche der Medizinaltarife berauben, sondern der FMH als ärztliche Selbstverwaltungsorganisation nicht 
heilbaren Schaden zufügen. Dies soll nicht heissen, dass die ÄK, bei akzeptiertem UV/MV/IVTarif, eine mit harten Konditionen versehene Rückweisung des KVG-Rahmenvertrags beschliessen kann und soll.

11. Es wäre eine Illusion zu glauben, die Tarifverhandlungen seien mit finalen Beschlüssen $\mathrm{zu}$ TARMED zu Ende. Tarif- bzw. besser: Preisverhandlungen werden in den kommenden Jahren unverändert im Zentrum gesundheitspolitischer Diskussionen stehen. Auf verhandlungstaktischer Ebene muss dies zur Einsicht führen, dass Verbesserung von Tarifstruktur und Durchsetzung ärztlicher Interessen - aktuell in Form des REProjektes - stetig und nachhaltig erfolgen müssen. Wer sich hier den grossen finalen Showdown herbeisehnt, könnte diesen als Dämmerung der weissen Götter erleben, die diese im Grunde nicht sein wollen und können.

\section{Fazit}

Aus diesen Ausführungen ergeben sich vor allem hinsichtlich kommender Vertragsverhandlungen folgende Punkte:

- Die Zeit praktisch ausschliesslich auf dem Milizsystem basierender, basisdemokratischer Verhandlungen gehört der Vergangenheit an. Die FMH hat in den letzten zwei Jahren eine Gruppe von Tariffachleuten aufgebaut, die auf der Verhandlungsebene wie der technischen «Ingenieursebene" professionell arbeiten. In diesem Zusammenhang wurde auch das Bezugsnetz und das Datenerfassungssystem konzipiert und implementiert, das die FMH von den Interessen (und im Klartext gesprochen: auch Erpressungen) Dritter unabhängig macht.

- Mit Sicherheit ist auch die Zeit kantonal-hoheitlicher Tarifverhandlungen vorbei. Solche Verhandlungen passen nicht mehr in eine Zeit, wo auch das schweizerische Sozialversicherungssystem dem europäischen Markt ausgesetzt ist und die Verhandlungskontrahenten ausschliesslich auf nationaler, bald auch einmal internationaler Ebene agieren. Diese Feststellung steht nicht im Gegensatz zu einer weiteren Feststellung: Der Widerstand der Ärzteschaft gegen Tarifdiktate müsste lokal-regional organisiert werden.
- In Zukunft dürfen die strategische, die Verhandlungsebene wie auch die operationell-technische Ebene nicht mehr vermischt werden. Insbesondere dürfen Akteure der Verhandlungsebene nicht mehr auf der strategischen oder der operationelltechnischen Ebene agieren.

- Neue Ziele können nur mit einem Schiff in Form eines finanziell gesicherten Projektes angesteuert werden, das über ein striktes, auch FMH-internes, Finanz- und Ablaufcontrolling verfügt. Dabei ist sicherzustellen, dass die Fahrt abgebrochen wird, wenn diese Voraussetzungen nicht mehr erfüllt sind bzw. das Ziel nicht mehr erreicht werden oder durch weitere implizite Zielsetzungen erweitert wurde. Der erste Prüfstein für diese im Grunde banal selbstverständliche Philosophie wird das Projekt RE sein.

- Wird der Ärzteschaft ein Projekt durch meist verdeckte und implizite politische Fremdbestimmung aus den Händen genommen, muss sie sich in $\mathrm{Zu}-$ kunft klar und unverzüglich von der Fortführung des entsprechenden Projektes distanzieren. Weitere Kooperationsbereitschaft wird in solchen Situationen nur als Schwäche und Anpassertum missverstanden. Dies muss insbesondere im Falle von Interventionen der PüW «avant la lettre» klar punktiert werden: der Vertrag gilt nur als erfüllt, wenn das Ergebnis der Verhandlungen integral umgesetzt und allfällige Interventionen der PüW gemeinsam akzeptiert werden.

- Tarifverhandlungen müssen als Kontinuum nach dem Prinzip des stetig den Stein höhlenden Tropfens begriffen und organisiert werden. TARMED, RE von TARMED sind nur Etappen auf einem endlosen Marsch, der bald in Gebiete wie Pauschalen/DRGs, Kosteneffizienz/Rationierung, Qualität des abrechnungsberechtigten Leistungserbringers u.a.m. führen werden. Die Strukturen der FMH, insbesondere aber auch ihr interner Entscheidungsprozess müssen in diesem Sinne ausgerichtet werden. Die FMH wird kaum mehr eine über Jahre andauernde, häufig auf persönlicher Ebene geführte und vor keinem Insult zurückschreckende Tarifrunde à la TARMED überstehen. Dies schon schlicht einfach deshalb nicht, weil sich für solche Arbeiten keine normalstrukturierten Menschen als Verantwortliche mehr finden lassen. Ich hoffe für meine Nachfolger, dass sie hier mehr Stil und Kollegialität erfahren dürfen, als mir zu erleben gegeben war. 